DOI: 10.12731/2070-7568-2018-2-111-128

УДК 330.4, 51-74

\title{
ОЦЕНКА ЭФФЕКТИВНОСТИ РАБОТЫ ПРЕДПРИЯТИЯ ПО РЕМОНТУ МАШИН И ОБОРУДОВАНИЯ НА ОСНОВЕ СТАТИСТИЧЕСКОГО АНАЛИЗА ИЗНОСА ДЕТАЛЕЙ
}

\section{Лажаунинкас Ю.В.}

В связи с экономическим кризисом, наблюдающимся во всех отраслях народного хозяйства, а также с недостаточным финансированием, в настоящее время происходит значительный спад производства сельскохозяйственной техники. Сельскохозяйственные предприятия не могут приобретать новую технику. В связи с этим возникает необходимость грамотного планирования применения имеющейся техники так, чтобы ее можно было использовать максимально эффективно, с минимальными затратами на ремонт и техническое обслуживание.

В экономической эффективности работь сельскохозяйственного предприятия немаловажную роль играет надежность техники, поэтому ее прогнозирование различными средствами позволяет снизить общие затраты. Правильная эксплуатащия и своевременный ремонт машин и оборудования позволяет повысить работоспособность, тем самым, снижая простои техники.

В настоящее время наблюдается довольно обостренная ситуация с проблемой надежности машин. Это, прежде всего, связано с все более возрастающей сложностью конструкции современных машин и механизмов, их многодетальностью, интенсификацией рабочих прочессов, тяжелыми условиями эксплуатации. Эффективность решения задачи замены или ремонта деталей, а следовательно, и вопрос об экономической эффективности работы ремонтного предприятия, находится в прямой зависимости от степени научнометодического сопровождения соответствующих мероприятий, одним из которых является проиесс оченки износа деталей. 
Цель исследования: исследовать эффективность работы предприятия по ремонту машин и оборудования на основе статистического анализа износа шейки под шарикоподшипник 208 вала первичной коробки передач МТ3-82, установить что в данном случае более эффективно - ремонт или замена рассматриваемой детали.

Методы исследования: методы статистического анализа.

Результаты: Получены наиболее информативные параметры, отражающие износ шейки под шарикоподшипник 208 вала первичной коробки передач МТ3-82. На основе проведенного анализа установлено, что, с экономической точки зрения, своевременное техническое обслуживание и текущий ремонт рассматриваемой детали является более эффективным, чем ее замена на новую.

Область применения результатов: результаты исследования могут быть использованы при оценке экономической эффективности работы предприятия по ремонту машин и оборудования.

Ключевые слова: экономическая эффективность; износ детали; статистический анализ; надежность машин.

\section{EVALUATION OF THE EFFECTIVENESS OF THE COMPANY REPAIR OF MACHINERY AND EQUIPMENT BASED ON STATISTICAL ANALYSIS OF WEAR}

\section{Lazhauninkas J.V.}

Due to the economic crisis in all sectors of the economy, as well as insufficient funding, there is now a significant decline in the production of agricultural machinery. Therefore, agricultural enterprises are unable to purchase new equipment. In this regard there is a need of competent planning of use of the available equipment so that it could be used most effectively, with the minimum expenses for repair and maintenance.

The economic efficiency of agricultural enterprises plays an important role in the reliability of equipment, so its forecasting by various means allows to reduce the total cost. Proper operation and timely re- 
pair of machinery and equipment can improve efficiency, thereby reducing downtime.

Currently, there is a rather acute situation with the problem of reliability of machines. This is primarily due to the increasing complexity of the design of modern machines and mechanisms, their large number, intensification of work processes, severe operating conditions. The efficiency of solving the problem of replacement or repair of parts, and therefore the question of the economic efficiency of the repair compa$n y$, is directly dependent on the degree of scientific and methodological support of relevant activities, one of which is the process of assessing the wear and tear of parts.

Purpose: to investigate efficiency of work of the enterprise for repair of cars and the equipment on the basis of the statistical analysis of wear of a neck under a ball-bearing of 208 shaft of a primary gearbox MTZ-82 to establish that in this case more effectively-repair or replacement of the considered detail.

Methodology in article: statistical analysis methods.

Results: Received the most informative parameters indicating the wear of the neck under the bearing 208 of the primary shaft of the gearbox MTZ-82. On the basis of the conducted analysis it is established that, from the economic point of view, timely maintenance and repair of the considered part is more effective than its replacement with a new one.

Practical implications: the results can be used to assess the economic efficiency of the enterprise for repair of machinery and equipment.

Keywords: economic efficiency; detail wear; statistical analysis; reliability of machines.

\section{Введение}

В настоящее время наблюдается значительный спад производства сельскохозяйственной техники $[6,16,18,20]$. Это, прежде всего, связано с экономическим кризисом, наблюдающимся во всех отраслях народного хозяйства, а также с недостаточным финансированием. В связи с этим сельскохозяйственные предприятия не могут приобретать новую технику. Поэтому очень важно грамотно планировать 
использование имеющейся техники с максимальной эффективностью и с минимальными затратами на ремонт и техническое обслуживание. Это, несомненно, будет способствовать повышению эффективности использования машин и оборудования, материальных и финансовых ресурсов, а также экономии рабочего времени.

Значительный вклад в изучение проблем экономической эффективности использования машинно-тракторного парка сельскохозяйственными предприятиями с учетом показателей надежности техники внесены работами Л.В. Канторовича [7, с. 71], Д.Б. Юдина [22, с. 103], Ю.М. Ермольева [4, с. 88], А.М. Гатаулина [2, с. 112], В.А. Кардаша [9, с. 38]. Но в указанной литературе не представлен подробный статистический анализ деталей или узлов сельскохозяйственной техники.

Проблема надежности машин, прежде всего, связана с все более возрастающей сложностью конструкции современных машин и механизмов, их многодетальностью, интенсификацией рабочих процессов, тяжелыми условиями эксплуатации.

Вопрос оценки износа деталей в современных условиях становится особенно актуальным $[5,10,13,17]$ и требует подробного изучения. С этой целью в данной работе проводится статистический анализ результатов, полученных в ходе сбора данных на одном из предприятий по ремонту сельскохозяйственной техники.

Сельскохозяйственные машины, выпускаемые современной промышленностью, обладают свойствами, определяющими их надежность и работоспособность. На их общее техническое состояние оказывает влияние ряд случайных факторов. Суммарное воздействие случайных факторов сказывается на интенсивности изнашивания деталей, частоте отказов и изменении показателей надежности техники в целом.

Срок службы сельскохозяйственной техники определяется износом их деталей - изменением размеров, форм, массы деталей, состоянием поверхностей вследствие изнашивания, усталостным и коррозийным разрушением и т.д. Скорость изнашивания деталей зависит от многих факторов: условий и режима работы, материала, из которого они изготовлены, состояния окружающей среды, типа 
смазки, температуры в точках сопряжения и т.д. Износ некоторых деталей можно обнаружить визуально. Износ других определяется замером зазоров, усилий на рычагах и приводах $[5,10,13,17]$.

Для того чтобы выявить потребность в ремонте или замене детали, необходимо спрогнозировать основные показатели ее надежности, зачастую без раскрытия влияния каждого случайного фактора. При выявленных объективных закономерностях и тенденциях можно повысить эффективность управления технологическими и производственными процессами, а также обеспечить определенную надежность работы сельскохозяйственной техники. Математический аппарат теории вероятностей и статистических методов анализа, а также теории надежности позволяет решать эти и другие задачи $[11,12,13,14]$.

\section{Материалы и методы}

В данной работе проводится статистический анализ износа деталей, а также производится оценка качества их восстановления. Расчеты проводятся для детали - вал первичной коробки передач МТ3-82 с контролируемым дефектом - износ шейки под шарикоподшипник 208.

\section{Результаты}

В результате экспериментального исследования были получены значения размеров изношенных деталей (таблица 1).

Таблийа 1.

\begin{tabular}{|c|c|c|c|c|c|c|c|c|c|}
\hline 39,961 & 39,931 & 39,928 & 39,954 & 39,945 & 39,958 & 39,942 & 39,932 & 39,959 & 39,938 \\
\hline 39,927 & 39,948 & 39,96 & 39,938 & 39,937 & 39,98 & 39,95 & 39,943 & 39,938 & 39,959 \\
\hline 39,966 & 39,975 & 39,945 & 39,92 & 39,931 & 39,935 & 39,956 & 39,959 & 39,962 & 39,953 \\
\hline 39,928 & 39,949 & 39,965 & 39,915 & 39,929 & 39,925 & 39,94 & 39,939 & 39,939 & 39,97 \\
\hline 39,947 & 39,922 & 39,941 & 39,972 & 39,943 & 39,954 & 39,953 & 39,959 & 39,924 & 39,977 \\
\hline 39,938 & 39,942 & 39,96 & 39,948 & 39,933 & 39,933 & 39,97 & 39,969 & 39,954 & 39,942 \\
\hline 39,952 & 39,954 & 39,928 & 39,961 & 39,96 & 39,956 & 39,94 & 39,96 & 39,919 & 39,951 \\
\hline 39,949 & 39,959 & 39,936 & 39,979 & 39,964 & 39,92 & 39,922 & 39,946 & 39,96 & 39,964 \\
\hline 39,972 & 39,953 & 39,948 & 39,942 & 39,966 & 39,949 & 39,947 & 39,946 & 39,937 & 39,964 \\
\hline 39,918 & 39,968 & 39,942 & 39,937 & 39,943 & 39,944 & 39,974 & 39,932 & 39,935 & 39,92 \\
\hline
\end{tabular}


116 Siberian Journal of Economics and Management, Vol. 7, No 2, 2018

Наименьший предельный размер вала составляет 39,992 мм.

Составим вариационный ряд величины износа (таблица 2).

Таблииа 2.

\begin{tabular}{|c|c|c|c|c|c|c|c|c|c|}
\hline $\begin{array}{c}\text { № } \\
\text { п/п }\end{array}$ & $\begin{array}{c}\text { Износ, } \\
\text { мм }\end{array}$ & $\begin{array}{c}\text { № } \\
\text { п/п }\end{array}$ & $\begin{array}{c}\text { Износ, } \\
\text { мм }\end{array}$ & $\begin{array}{c}\text { № } \\
\text { п/п }\end{array}$ & $\begin{array}{c}\text { Износ, } \\
\text { мм }\end{array}$ & $\begin{array}{c}\text { № } \\
\text { п/п }\end{array}$ & $\begin{array}{c}\text { Износ, } \\
\text { мм }\end{array}$ & $\begin{array}{c}\text { № } \\
\text { п/п }\end{array}$ & $\begin{array}{c}\text { Износ, } \\
\text { мм }\end{array}$ \\
\hline 1. & 0,012 & 21. & 0,032 & 41. & 0,04 & 61. & 0,050 & 81. & 0,059 \\
\hline 2. & 0,013 & 22. & 0,032 & 42. & 0,041 & 62. & 0,050 & 82. & 0,060 \\
\hline 3. & 0,015 & 23. & 0,032 & 43. & 0,042 & 63. & 0,050 & 83. & 0,060 \\
\hline 4. & 0,017 & 24. & 0,032 & 44. & 0,043 & 64. & 0,050 & 84. & 0,061 \\
\hline 5. & 0,018 & 25. & 0,032 & 45. & 0,043 & 65. & 0,051 & 85. & 0,061 \\
\hline 6. & 0,020 & 26. & 0,033 & 46. & 0,043 & 66. & 0,052 & 86. & 0,063 \\
\hline 7. & 0,020 & 27. & 0,033 & 47. & 0,044 & 67. & 0,052 & 87. & 0,064 \\
\hline 8. & 0,022 & 28. & 0,033 & 48. & 0,044 & 68. & 0,053 & 88. & 0,064 \\
\hline 9. & 0,022 & 29. & 0,033 & 49. & 0,044 & 69. & 0,053 & 89. & 0,064 \\
\hline 10. & 0,023 & 30. & 0,033 & 50. & 0,045 & 70. & 0,054 & 90. & 0,065 \\
\hline 11. & 0,024 & 31. & 0,034 & 51. & 0,045 & 71. & 0,054 & 91. & 0,067 \\
\hline 12. & 0,026 & 32. & 0,036 & 52. & 0,046 & 72. & 0,054 & 92. & 0,068 \\
\hline 13. & 0,026 & 33. & 0,036 & 53. & 0,046 & 73. & 0,054 & 93. & 0,070 \\
\hline 14. & 0,027 & 34. & 0,038 & 54. & 0,047 & 74. & 0,055 & 94. & 0,070 \\
\hline 15. & 0,028 & 35. & 0,038 & 55. & 0,047 & 75. & 0,055 & 95. & 0,072 \\
\hline 16. & 0,028 & 36. & 0,038 & 56. & 0,048 & 76. & 0,055 & 96. & 0,072 \\
\hline 17. & 0,028 & 37. & 0,038 & 57. & 0,049 & 77. & 0,056 & 97. & 0,072 \\
\hline 18. & 0,030 & 38. & 0,039 & 58. & 0,049 & 78. & 0,057 & 98. & 0,073 \\
\hline 19. & 0,031 & 39. & 0,039 & 59. & 0,049 & 79. & 0,057 & 99. & 0,074 \\
\hline 20. & 0,031 & 40. & 0,039 & 60. & 0,050 & 80. & 0,059 & 100. & 0,077 \\
\hline
\end{tabular}

Составим статистический ряд распределения (таблица 3), для чего разобьем вариационный ряд на $n=10$ интервалов. Длина каждого интервала вычисляется по формуле $h=\frac{I_{\max }-I_{\min }}{n}$, тогда $h \approx 0,0065$ мм. Наблюдаемая (опытная) вероятность в каждом интервале определяется по формуле $\bar{p}_{i}=\frac{m_{i}}{N}$, где $m_{i}-$ наблюдаемая частота, $N=100$ - объем выборки. Значения накопленных опытных вероятностей определяются суммированием вероятностей по интервалам: $\bar{F}(I)=\sum \bar{p}_{i}$. 
Таблииа 3.

\begin{tabular}{|l|c|c|c|c|c|c|c|c|c|c|}
\hline $\begin{array}{l}\text { Границы } \\
\text { интервала, мм }\end{array}$ & $\begin{array}{c}0,012 \\
-\end{array}$ & $\begin{array}{c}0,019 \\
-\end{array}$ & $\begin{array}{c}0,025 \\
-\end{array}$ & $\begin{array}{c}0,0315 \\
-\end{array}$ & $\begin{array}{c}0,038 \\
-\end{array}$ & $\begin{array}{c}0,0445 \\
-\end{array}$ & $\begin{array}{c}0,051 \\
-\end{array}$ & $\begin{array}{c}0,0575 \\
-\end{array}$ & $\begin{array}{c}0,064 \\
-\end{array}$ & $\begin{array}{c}0,0705 \\
- \\
0,0315\end{array}$ \\
\hline $\begin{array}{l}\text { Середина } \\
\text { интервала, мм }\end{array}$ & 0,015 & 0,022 & 0,028 & 0,038 & 0,041 & 0,048 & 0,054 & 0,061 & 0,067 & 0,074 \\
\hline $\begin{array}{l}\text { Опытная } \\
\text { частота } m_{i}\end{array}$ & 5 & 6 & 9 & 17 & 12 & 16 & 14 & 10 & 5 & 6 \\
\hline $\begin{array}{l}\text { Опытная } \\
\text { вероятность } \bar{p}_{i}\end{array}$ & 0,05 & 0,06 & 0,09 & 0,17 & 0,12 & 0,16 & 0,14 & 0,1 & 0,05 & 0,06 \\
\hline $\begin{array}{l}\text { Накопленная } \\
\text { опытная вероят- } \\
\text { ность } \bar{F}(I)\end{array}$ & 0,05 & 0,11 & 0,2 & 0,37 & 0,49 & 0,65 & 0,79 & 0,89 & 0,94 & 1 \\
\hline
\end{tabular}

Определим основные числовые характеристики износов. К ним относятся среднее значение износа (математическое ожидание), среднее квадратическое отклонение и коэффициент вариации. Среднее значение износа характеризует центр группирования случайной величины [15]. Среднее квадратическое отклонение и коэффициент вариации являются характеристиками рассеивания случайной величины от величины среднего износа.

Среднее значение износа вычислим по известной формуле $\bar{I}=\sum_{i=1}^{n} I_{c i} \cdot \bar{p}_{i}$, где $I_{c i}-$ середина $i$-го интервала, $\bar{p}_{i}-$ опытная вероятность в $i$-ом интервале. Тогда $\bar{I} \approx 0,045$ мм.

Среднее квадратическое отклонение $\sigma_{I}=\sqrt{\sum_{i=1}\left(I_{c i}-\bar{I}\right)^{2} \cdot p_{i}=}$ $=0,016$ мм. Коэффициент вариации рассчитаем по формуле $V=\frac{\sigma}{I-I_{\text {см }}}$, где $I_{\text {см }}-$ смещение начала рассеяния, при $N>25$ $I_{\text {см }}=I_{1}-0,5 \cdot h=0,0012-0,5 \cdot 0,0065=0,0088$ мм. Тогда $V=$ $=\frac{0,016}{0,045-0,0088}=0,43$.

Величина коэффициента вариации влияет на выбор теоретического закона распределения. Практика изучения износов сельскохозяйственной техники показывает, что износ, как случайная величи- 
на лучше всего описываются законами нормального распределения (ЗНР) и распределения Вейбулла (ЗРВ).

Поскольку $V=0,43$ попадает в интервал от 0,3 до 0,5 , то выбирается тот закон распределения, который обеспечивает лучшее совпадение с распределением опытной информацией. Для этого необходимо рассчитаем дифференциальную и интегральную функции распределения износа детали по ЗНР и ЗРВ, с последующей проверкой правдоподобия каждого из них по одному из критериев согласия и примем соответствующее решение.

Для нормального закона распределения. Так как при составлении статистического ряда (см. таблицу 3) были вычислены не статистические плотности функции распределения $\bar{f}(I)$, а опытные вероятности попадания наблюдений в $i$-й интервал $\bar{p}_{i}$, то для обеспечения сравнимости распределений вычислим теоретические вероятности этих же событий по зависимости: $p\left(I_{c i}\right)=\frac{h}{\sigma_{I}} f_{0}\left(z_{i}\right)$, где $h-$ длина интервала, принятая при построении статистического ряда; $z_{i}=\frac{I_{c i}-\bar{I}}{\sigma_{I}}-$ квантиль нормального распределения, значение которого вычислено для середины $i$-го интервала $I_{c i} ; f_{0}\left(z_{i}\right)=f_{0}\left(\frac{I_{c i}-\bar{I}}{\sigma_{I}}\right)-$ значение центрированной и нормированной плотности распределения, которые берутся из известных таблиц (при этом $f_{0}(-z)=f_{0}(z)$ ); $n$ - число интервалов, принятое при составлении статистического ряда.

Значения теоретических вероятностей запишем в таблицу 4.

Интегральная функция распределения вычислим по формуле: $F(I)=F_{0}\left(z_{i}\right)$, где $z_{i}=\frac{I_{c i}-\bar{I}}{\sigma_{I}}-$ квантиль нормального распределения, значение которого вычислено для конца $i$-го интервала; $F_{0}\left(z_{i}\right)-$ значение интегральной функции нормального распределения (при этом $\left.F_{0}(-z)=1-F_{0}(z)\right)$. Полученные значения функции запишем в таблицу 4.

Используя значение функции распределения, определим теоретическое число интересующих нас событий (число отказов в $i$-м 
интервале) по формуле: $m_{T_{i}}=\left(F\left(I_{k i}\right)-F\left(I_{k(i-1)}\right) N\right.$, где $N$ - объем выборки; $F\left(I_{k i}\right), F\left(I_{k(i-1)}\right)$ - интегральные функции в конце и в начале $i$-го интервала.

Определим значения теоретических частот для каждого интервала и занесем полученные значения в таблицу 4 .

Для закона распределения Вейбулла. Вычислим не $f(I)$, а теоретические вероятности попадания случайной величины в $i$-й интервал. $p\left(I_{c i}\right)=\frac{h}{a} \cdot f_{i}\left(\frac{I_{c i}-I_{\text {см }}}{a}, b\right)$, где $h$ - длина интервала; $a-$ ресурсная характеристика, параметр масштаба ЗРВ; $b$ - параметр формы; $I_{\text {см }}$ - смещение начала рассеяния; $f_{i}\left(\frac{I_{c i}-I_{\text {см }}}{a}, b\right)$ - табулированное значение дифференциальной функции.

Параметры ЗРВ определяются в зависимости от коэффициента вариации. Так как $V=0,43$, то $\mathrm{b}=2,5, K_{b}=0,89, C_{b}=0,38$.

Расчет $f\left(I_{c i}\right)$ для ЗРВ проведем для каждого интервала и полученные данные запишем в таблицу 4.

Функция распределения Вейбулла имеет вид: $F\left(I_{k i}\right)=$ $=1-\exp \left(-\left(\frac{I_{k i}-I_{c \mathrm{M}}}{a}\right)^{b}\right)$. Данная функция зависит от двух аргументов - от параметра $b$ и обобщенного параметра $\left(\frac{I_{k i}-I_{\mathrm{cm}}}{a}\right)$. Полученные значения внесем в таблицу 4.

Используя значение функции распределения, вычислим теоретическое число интересующих нас событий $\mathrm{m}_{\mathrm{T}_{\mathrm{i}}}=\left(\mathrm{F}\left(\mathrm{I}_{\mathrm{ki}}\right)-\mathrm{F}\left(\mathrm{I}_{\mathrm{k}(\mathrm{i}-1)}\right) \mathrm{N}\right.$, где $\mathrm{N}$ - объем выборки; $\mathrm{F}\left(\mathrm{I}_{\mathrm{ki}}\right), \mathrm{F}\left(\mathrm{I}_{\mathrm{k}(\mathrm{i}-1)}\right)$ - интегральные функции в конце и в начале і-го интервала. Полученные значения внесем в таблицу 4.

Таблициа 4.

\begin{tabular}{|c|c|c|c|c|c|c|c|c|c|c|}
\hline $\begin{array}{c}\text { Границы интервала, } \\
\text { мм }\end{array}$ & $\begin{array}{c}0,012 \\
- \\
0,019\end{array}$ & $\begin{array}{c}0,019 \\
- \\
0,025\end{array}$ & $\begin{array}{c}0,025 \\
- \\
0,031\end{array}$ & $\mid \begin{array}{c}0,031 \\
- \\
0,038\end{array}$ & $\begin{array}{c}0,038 \\
- \\
0,044\end{array}$ & $\begin{array}{c}0,044 \\
- \\
0,051\end{array}$ & $\mid \begin{array}{c}0,051 \\
- \\
0,057\end{array}$ & $\begin{array}{c}0,057 \\
- \\
0,064\end{array}$ & $\begin{array}{c}0,064 \\
- \\
0,070\end{array}$ & $\begin{array}{c}0,070 \\
- \\
0,077\end{array}$ \\
\hline $\begin{array}{c}\text { Середина интервала, } \\
\text { мм }\end{array}$ & 0,015 & 0,022 & 0,028 & 0,038 & 0,041 & 0,048 & 0,054 & 0,061 & 0,067 & 0,074 \\
\hline $\begin{array}{c}\text { Опытная частота } \\
m_{i}\end{array}$ & 5 & 6 & 9 & 17 & 12 & 16 & 14 & 10 & 5 & 6 \\
\hline
\end{tabular}


120 Siberian Journal of Economics and Management, Vol. 7, No 2, 2018

Окончание табл. 4.

\begin{tabular}{|c|c|c|c|c|c|c|c|c|c|c|c|c|}
\hline \multirow{3}{*}{ 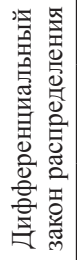 } & \multicolumn{2}{|c|}{$\begin{array}{c}\text { Опытная } \\
\text { вероятность } \bar{p}\left(I_{c i}\right)\end{array}$} & 0,05 & 0,06 & 0,09 & 0,17 & 0,12 & 0,16 & 0,14 & 0,1 & 0,05 & 0,06 \\
\hline & \multirow{2}{*}{$\begin{array}{c}\text { Теоретическая } \\
\text { вероятность } \\
p\left(I_{c i}\right)\end{array}$} & $\hat{m}$ & 0,0248 & 0,0505 & 0,0917 & 0,1329 & 0,1629 & 0,1625 & 0,1409 & 0,0987 & 0,062 & 0,0284 \\
\hline & & $\stackrel{m}{\frac{m}{m}}$ & 0,0307 & 0,0693 & 0,1095 & 0,1409 & 0,1574 & 0,1519 & 0,1262 & 0,0896 & 0,0554 & 0,0313 \\
\hline \multirow{3}{*}{ 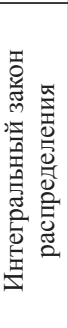 } & \multicolumn{2}{|c|}{$\begin{array}{c}\text { Накопленная } \\
\text { опытная вероят- } \\
\text { ность } \\
\bar{F}(I)=\sum \bar{p}\left(I_{c i}\right)\end{array}$} & 0,05 & 0,11 & 0,2 & 0,37 & 0,49 & 0,65 & 0,79 & 0,89 & 0,94 & 1 \\
\hline & \multirow{2}{*}{$\begin{array}{c}\text { Функция } \\
\text { распределения } \\
F_{I}=F\left(I_{k i}\right)\end{array}$} & $\stackrel{m}{M}$ & 0,0238 & 0,0756 & 0,187 & 0,308 & 0,48 & 0,659 & 0,796 & 0,89 & 0,977 & 0,98 \\
\hline & & $\frac{n}{\text { ले }}$ & 0,031 & 0,093 & 0,194 & 0,348 & 0,495 & 0,659 & 0,777 & 0,878 & 0,934 & 0,972 \\
\hline \multirow{2}{*}{\multicolumn{2}{|c|}{$\begin{array}{c}\text { Теоретическая } \\
\text { частота } m_{T i}\end{array}$}} & $\hat{m}$ & 2,38 & 5,18 & 11,14 & 12,15 & 17,18 & 17,96 & 13,65 & 9,36 & 8,75 & 0,25 \\
\hline & & $\frac{m}{m}$ & 3,1 & 6,31 & 10,06 & 15,42 & 14,67 & 16,37 & 11,79 & 10,14 & 5,63 & 3,75 \\
\hline
\end{tabular}

Проверим правдоподобие (сходимость) опытного и теоретического законов распределения по критерию согласия Пирсона. Для этого рассчитаем величину $\chi^{2}=\sum_{i=1}^{n} \frac{\left(m_{i}-m_{T_{i}}\right)^{2}}{m_{T_{i}}}$, где $m_{i}$ - опытная частота попадания случайной величины в $i$-тый интервал статистического ряда; $m_{T i}$ - теоретическая частота в $i$-том интервале.

Значение критерия для ЗРВ $\chi^{2}=5,45$, а для Н3Р $\chi^{2}=5,64$. число степеней свободы $\mathrm{k}=\mathrm{n}-(\mathrm{m}+1)=10-(2+1)=7$, где $n$ - число интервалов статистического ряда, а $m$ - число параметров теоретического закона распределения (для НЗР и ЗРВ $\mathrm{m}=2$ ); приняты уровень значимости (вероятность необоснованного отклонения гипотезы) $\alpha=0,05$. Необходимо выбрать теоретический закон распределения, наиболее адекватный распределению статистической информации.

По известным таблицам определим критические значения критерия Пирсона. Для ЗРВ $\chi^{2}(a, k)=12,6$, для НЗР $\chi^{2}(a, k)=11,1$. В данном случае для обоих законов выполняется $\chi^{2}<\chi^{2}(a, k)$, поэтому выдвинутая гипотеза о сходимости опытного с теорети- 
ческими распределениями с вероятностью $p=1-a=0,95$ не отвергается.

Для принятия окончательного решения определим вероятность подтверждения проверяемого теоретического закона распределения. Для ЗРВ Р $=48,68 \%$, для НЗР Р $=33,99 \%$. Следовательно, в данном случае принимаем гипотезу о том, что анализируемая статистическая информация с достаточной степенью достоверности подчиняется закону распределения Вейбулла [3, с. 382,19 , с. 157$]$.

Определим доверительный интервал исследуемого значения износа: $\left(\bar{I}-I_{\text {см }}\right) \cdot \sqrt[b]{r_{3}}+I_{\text {см }}<m_{t}<\left(\bar{I}-I_{\text {см }}\right) \cdot \sqrt[b]{r_{1}}+I_{\text {см }}$ где $I_{\text {см }}=0,0088$, $r_{1}=1,23$ и $r_{3}=0,83$ - коэффициенты распределения Вейбулла и $\beta=0,95$ выбирается из таблицы. Получаем нижнюю и правую границы доверительного интервала: $I_{\beta 1}=0,0424$ мм и $I_{\beta 2}=0,0481$ мм соответственно.

То есть с вероятностью $\beta=0,95$ можно утверждать, что исследуемое значение износа детали находится в интервале от 0,0424 до 0,0481 мм.

Определим относительную ошибку точности оценки износа, которая позволяет корректно сравнивать объекты и по разнородным показателям. $\varepsilon=\frac{I_{\beta_{0}}-\bar{I}}{\bar{I}-I_{\text {см }}} \cdot 100 \%$, где $I_{\beta 0}-$ верхняя граница изменения среднего значения износа, установленного с вероятностью $\beta_{0}=0,95$. Тогда $\varepsilon=\frac{0,0481-0,045}{0,045-0,0088} \cdot 100 \%=4,9 \%$. Максимально допустимая ошибка не превышает $20 \%$.

Определим число годных и требующих восстановления деталей. Допустимые износы детали при сопряжении с новыми $I_{\text {дн }}$ и быв-

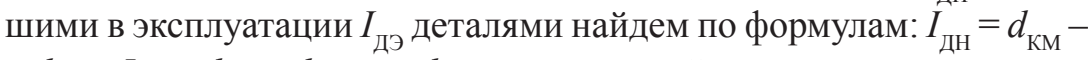
$-d_{\text {дн }}$ и $I_{\text {дэ }}=d_{\text {км }}-d_{\text {дэ }}$ где $d_{\text {дн }}-$ допустимый размер вала при сопряжении его с новыми деталями; $d_{\text {дэ }}-$ допустимый размер вала при сопряжении его с деталями, бывшими в эксплуатации; $d_{\text {км }}-$ наименьший предельный размер вала. $I_{\text {дн }}=39,992-39,970=0,022$ мм и $I_{\text {дэ }}=39,992-39,990=0,002$ мм. 


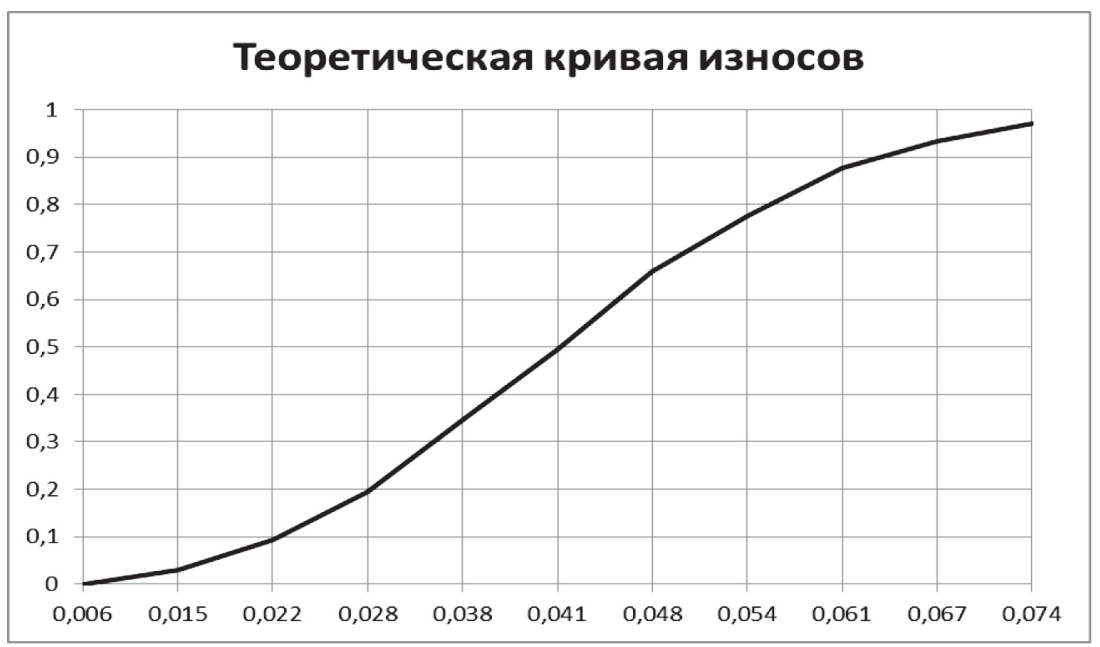

Рис. 1. Теоретическая кривая износов

Найдем количество годных деталей, для этого определим вероятность $p_{\text {гн }}$, того что детали окажутся годными (их восстановление не потребуется) при условии их сборки с новыми сопрягаемыми деталями [17]. Значение допустимого износа $I_{\text {дн }}=0,022$ отложим по оси абсцисс (рис. 1) и восстановим перпендикуляр до пересечения с теоретической кривой износов $F(I)$. Полученную точку проектируем на ось ординат. Получим искомую вероятность $p_{\Gamma н}=0,093$. Тогда процент годных без ремонта деталей составляет 9,3\%. Аналогично, для значения $I_{\text {дэ }}=0,002$ определяем процент годных деталей при сопряжении их с деталями, бывшими в эксплуатации, получаем $0 \%$.

Итак, коэффициент годности анализируемых деталей составляет 0,093, а коэффициент восстановления деталей 0,907.

Таким образом, по значениям вычисленных коэффициентов заключаем, что необходимо более тщательно составлять план работы ремонтного предприятия по анализируемой детали.

Полученные значения свидетельствуют о том, что ремонтное предприятие неэффективно обслуживает клиентов, не позволяя им минимизировать затраты. Процент годных деталей слишком мал, а это говорит о том, что не вовремя было проведено техническое обслу- 
живание техники, не был произведен необходимый ремонт анализируемой детали. Поэтому в сложившейся ситуации требуется замена шарикоподшипника 208 вала первичной коробки передач МТ3-82.

Стоимость шарикоподшипника 208 вала первичной коробки передач МТЗ-82 порядка 200 руб. Замена шарикоподшипника 208 вала первичной коробки передач МТ3-82 составляет порядка 3000 руб. В то время как своевременное техническое обслуживание техники с текущим ремонтом указанной детали составляет порядка 1000 руб. В некоторых случаях достаточно смазать подшипник, чтобы нормализовать его работу и устранить шум в КПП. Если игнорировать признаки неисправности подшипника, то впоследствии может потребоваться серьезный ремонт сцепления или коробки передач. Сравнивая приведенные стоимости, делаем вывод, что эффективнее проводить своевременный текущий ремонт.

\section{Заключение}

В экономической эффективности работы сельскохозяйственного предприятия немаловажную роль играет надежность техники, поэтому ее прогнозирование различными средствами позволяет снизить общие затраты. Правильная эксплуатация и своевременный ремонт машин и оборудования позволяет повысить работоспособность, тем самым, снижая простои техники.

В данной работе проведен статистический анализ износа шейки под шарикоподшипник 208 вала первичной коробки передач МТ382. Получены наиболее информативные параметры, отражающие износ рассматриваемой детали. На основе проведенного анализа сделан вывод о том, что, с экономической точки зрения, своевременное техническое обслуживание и текущий ремонт рассматриваемой детали является более эффективным, чем ее замена на новую.

\section{Список литературы}

1. Гатаулин А.М. Издержки производства сельскохозяйственной продукции (методология измерения и пути снижения). М.: «Экономика», 1983. $184 \mathrm{c.}$ 
2. Гатаулин А.М. Экономико-математические методы в планировании сельскохозяйственного производства / А.М. Гатаулин, Г.В. Гаврилов, Л.А. Харитонова. М.: «Колос», 1976. 223 с.

3. Гмурман В.Е. Теория вероятностей и математическая статистика: учебное пособие для бакалавров. Изд. 12-е. М.: «Юрайт», 2014. 479 с.

4. Ермольев Ю.М., Ястремский А.И. Стохастические модели и методы в экономическом планировании. М.: «Наука», 1979. 255 с.

5. Ерохин М.Н. Оценка износа крестовин шарниров типа CR115, применяемых в тракторах JOHN DEERE / M.H. Ерохин, А.Г. Пастухов, Е.П. Тимашов // Труды ГОСНИТИ. 2017. Т. 126. С. 14-21.

6. Кайшев В.Г., Алпатов А.В. Сельскохозяйственная техника в России: рыночные позиции внутреннего производства на фоне внешнеэкономических отношений // Экономика сельскохозяйственных и перерабатывающих предприятий. 2017. № 4. С. 19-27.

7. Канторович Л.В., Горстко А.Б. Математическое оптимальное программирование в экономике. М.: «Знание», 1968. 95 с.

8. Кардаш В.А. Модели управления производственно-экономическими процессами в сельском хозяйстве. М.: «Колос», 1981. 183 с.

9. Кардаш В.А., Рапопорт Э.О. Моделирование экономических процессов в сельском хозяйстве. Новосибирск: «Наука. Сиб. Отделение», 1979. $157 \mathrm{c}$.

10. Королев А.Е. Расчетно-экспериментальный метод определения допускаемого износа двигателей // Вестник Государственного аграрного университета Северного Зауралья. 2016. № 1 (32). С. 135-139.

11. Кочегарова О.С., Лажаунинкас Ю.В. АВС-анализ как средство оптимизации решения задач прикладной статистики // Наука Красноярья. 2016. № 3-3 (26). С. 101-109.

12. Кочегарова О.С., Лажаунинкас Ю.В. Прогнозирование экономических процессов методами корреляционно-регрессионного анализа // О вопросах и проблемах современных математических и естественных наук: сб. науч. тр. Челябинск, 2015. С. 9-12.

13. Кунтурова Н.Б., Кунтуров С.А. Расчет совокупного износа деталей механизмов машин // Труды Военно-космической академии им. А.Ф. Можайского. 2015. № 648. С. 153-156. 
14. Лажаунинкас Ю.В. Т-статистика в Excel // Аграрная наука в XXI веке: проблемы и перспективы Сборник статей Всероссийской научно-практической конференции. Саратов, 2017. С. 154-159.

15. Лажаунинкас Ю.В., Кочегарова О.С. Методика проведения статистической обработки экспериментальных данных с использованием табличного процессора Excel // Фундаментальные и прикладные исследования в высшей аграрной школе. Под ред. М.В. Муравьевой и Г.Н. Камышовой. Саратов, 2014. С. 34-36.

16.Нечаев В.Н. Анализ и развитие рынка сельскохозяйственной техники как направление обеспечения продовольственной безопасности / В.Н. Нечаев, М.Л. Нечаева, Е.В. Ганебных // Международный технико-экономический журнал. 2017. № 4. С. 21-30.

17. Осипов С.Н., Поздняков Д.А. Об оценке физического износа элементов технических устройств // Наука и техника. 2015. №2. С. 23-30.

18. Семененко И.В., Снимщикова И.В. Оценка состояния рынка сельскохозяйственной техники: российский и зарубежный аспект // Вестник АПК Ставрополья. 2015. № 2 (18). С. 303-307.

19. Сидняев Н.И. Теория планирования эксперимента и анализ статистических данных: учеб. пособие для магистров. М.: «Юрайт», 2012. 399 с.

20. Тиндова М.Г. Анализ производства тракторов и сельскохозяйственной техники // Агропродовольственная экономика. 2017. № 5. С. 83-90.

21. Юдин Д.Б. Математические методы управления в условиях неполной информации. М.: «Советское радио», 1974. 399 с.

22. Юдин Д.Б. Экстремальные модели в экономике. М.: «Экономика», $1979.287 \mathrm{c}$.

\section{References}

1. Gataulin A.M. Izderzhki proizvodstva sel'skokhozyaystvennoy produktsii (metodologiya izmereniya i puti snizheniya) [Agricultural production costs (measurement methodology and ways of reducing)]. Moscow, «Ekonomika», 1983. 184 p.

2. Gataulin A.M. Ekonomiko-matematicheskie metody v planirovanii sel'skokhozyaystvennogo proizvodstva [Economic and mathematical methods in agricultural production planning]. Moscow, «Kolos», 1976. 223 p. 
3. Gmurman V.E. Teoriya veroyatnostey i matematicheskaya statistika: uchebnoe posobie dlya bakalavrov [Probability theory and mathematical statistics: a textbook for bachelors]. Moscow, «Yurayt», 2014. 479 p.

4. Ermol'ev Yu.M. Stokhasticheskie modeli i metody v ekonomicheskom planirovanii [Stochastic models and methods in economic planning]. Moscow, «Nauka», 1979. 255 p.

5. Erokhin M.N. Otsenka iznosa krestovin sharnirov tipa CR115, primenyaemykh v traktorakh JOHN DEERE [Evaluation of wear of CR 115 type cross joints used in JOHN DEERE tractors]. Trudy GOSNITI. 2017. Vol. 126, pp. 14-21.

6. Kayshev V.G., Alpatov A.V. Sel'skokhozyaystvennaya tekhnika v Rossii: rynochnye pozitsii vnutrennego proizvodstva na fone vneshneekonomicheskikh otnosheniy [Agricultural machinery in Russia: market positions of domestic production against foreign economic relations]. Ekonomika sel'skokhozyaystvennykh i pererabatyvayushchikh predpriyatiy. 2017. Vol 4, pp. 19-27.

7. Kantorovich L.V., Gorstko A.B. Matematicheskoe optimal'noe programmirovanie v ekonomike [Optimal mathematical programming in the economics]. Moscow, «Znanie», 1968. 95 p.

8. Kardash V.A. Modeli upravleniya proizvodstvenno-ekonomicheskimi protsessami $v$ sel'skom khozyaystve [Models of management of production and economic processes in agriculture]. Moscow, «Kolos», 1981. $183 \mathrm{p}$.

9. Kardash V.A., Rapoport E.O. Modelirovanie ekonomicheskikh protsessov $v$ sel'skom khozyaystve [Modeling of economic processes in agriculture]. Novosibirsk: «Nauka. Sib. Otdelenie», 1979. 157 p.

10. Korolev A.E. Raschetno-eksperimental'nyy metod opredeleniya dopuskaemogo iznosa dvigateley [Calculation and experimental method for determining the permissible engine wear]. Vestnik Gosudarstvennogo agrarnogo universiteta Severnogo Zaural'ya. 2016. no. 1 (32), pp. 135-139.

11. Kochegarova O.S., Lazhauninkas Yu.V. ABS-analiz kak sredstvo optimizacii reshenija zadach prikladnoj statistiki [ABC-analysis as a means to optimize the solution of tasks applied statistics]. Nauka Krasnojar 'ja. 2016. no. 3-3 (26), pp. 101-109. 
12. Kochegarova O.S., Lazhauninkas Yu.V. Prognozirovanie ekonomicheskih processov metodami korreljacionno-regressionnogo analiza [Forecasting of economic processes by methods of correlation and regression analysis]. Chelyabinsk, 2015, pp. 9-12.

13. Kunturova N.B., Kunturov S.A. Raschet sovokupnogo iznosa detaley mekhanizmov mashin [Calculation of total wear and tear of machine parts]. Trudy Voenno-kosmicheskoy akademii im. A.F. Mozhayskogo. 2015. no. 648, pp. 153-156.

14. Lazhauninkas Yu.V. T-statistika v Excel [T-statistics in Excel]. Agrarnaya nauka v XXI veke: problemy i perspektivy Sbornik statey Vserossiyskoy nauchno-prakticheskoy konferentsii. Saratov. 2017, pp. 154-159.

15. Lazhauninkas Yu.V., Kochegarova O.S. Metodika provedenija statisticheskoj obrabotki jeksperimental'nyh dannyh s ispol'zovaniem tablichnogo processora Excel [The methodology of statistical processing of experimental data using the Excel spreadsheet processor]. Saratov, 2014, pp. 34-36.

16. Nechaev V.N. Analiz i razvitie rynka sel'skokhozyaystvennoy tekhniki kak napravlenie obespecheniya prodovol'stvennoy bezopasnosti [Analysis and development of the market of agricultural machinery as a direction of food security]. Mezhdunarodnyy tekhniko-ekonomicheskiy zhurnal. 2017. Vol 4, pp. 21-30.

17. Osipov S.N., Pozdnyakov D.A. Ob otsenke fizicheskogo iznosa elementov tekhnicheskikh ustroystv [About assessment of physical wear of elements of technical devices]. Nauka i tekhnika. 2015. no. 2, pp. 23-30.

18. Semenenko I.V., Snimshchikova I.V. Otsenka sostoyaniya rynka sel'skokhozyaystvennoy tekhniki: rossiyskiy i zarubezhnyy aspekt [Assessing the state of the agricultural machinery market: Russian and foreign aspects]. Vestnik APK Stavropol'ya. 2015. Vol. 2 (18), pp. 303-307.

19. Sidnyaev N.I. Teoriya planirovaniya eksperimenta $i$ analiz stati-sticheskikh dannykh : ucheb. posobie dlya magistrov [Theory of experiment planning and statistical data analysis: studies. handbook for masters]. Moscow, «Yurayt», 2012. 399 p.

20. Tindova M.G. Analiz proizvodstva traktorov i sel'skokhozyaystvennoy tekhniki [Analysis of tractors and agricultural machinery production]. Agroprodovol 'stvennaya ekonomika. 2017. Vol. 5, pp. 83-90. 
21. Yudin D.B. Matematicheskie metody upravleniya v usloviyakh nepol-noy informatsii [Mathematical methods of control in conditions of incomplete information]. Moscow, «Sovetskoe radio», 1974. 399 p.

22. Yudin D.B. Ekstremal'nye modeli v ekonomike [Extreme models in economics]. Moscow, «Ekonomika», 1979. 287 p.

\section{ДАННЫЕ ОБ АВТОРЕ}

Лажаунинкас Юлия Владимировна, доцент кафедры «Экономическая кибернетика», кандидат педагогических наук ФГБОУ ВО Саратовский государственный аграрный университет имени Н.И. Вавилова

Театральная пл., 1, г. Саратов, 410012, Российская Федераџия lazhauninkas@yandex.ru

\section{DATA ABOUT THE AUTHOR}

Lazhauninkas Julia Vladimirovna, Associate Professor «Economic Cybernetics», Candidate of Pedagogical Sciences Saratov State Agrarian University

1, Teatral'naya Sq., Saratov, 410012, Russian Federation lazhauninkas@yandex.ru

SPIN-code: 8735-6760

ORCID: 0000-0002-8018-7818 